Discrete Comput Geom 37:629-640 (2007)

DOI: $10.1007 / \mathrm{s} 00454-007-1315-\mathrm{x}$

\title{
The Genus of a Digital Image Boundary Is Determined by Its Foreground, Background, and Reeb Graphs
}

\author{
Lowell Abrams ${ }^{1}$ and Donniell E. Fishkind ${ }^{2}$ \\ ${ }^{1}$ Department of Mathematics, George Washington University, \\ Washington, DC 20052, USA \\ labrams@gwu.edu \\ ${ }^{2}$ Department of Applied Mathematics and Statistics, Johns Hopkins University, \\ Baltimore, MD 21218-2682, USA \\ fishkind@ams.jhu.edu
}

\begin{abstract}
We prove that the genus of the boundary of a digital image is precisely half of the sum of the cycle ranks of three particular graphs: the "foreground graph" and "background graph," which capture topological information about the digital image and its complement, respectively, and the Reeb graph, relative to the natural height function, associated with the digital image's boundary. We prove several additional results, including a characterization of when the cycle rank of the Reeb graph fails to equal the genus of the digital image's boundary (which can happen by virtue of the failure of the natural height function on the boundary of the digital image to be a Morse function).
\end{abstract}

\section{Overview}

A digital image $\mathcal{I}$ is a union of unit cubes in $\mathbb{R}^{3}$, each unit cube being of the form $\left[i-\frac{1}{2}, i+\frac{1}{2}\right] \times\left[j-\frac{1}{2}, j+\frac{1}{2}\right] \times\left[k-\frac{1}{2}, k+\frac{1}{2}\right]$ for integers $i, j, k$. We require, moreover, that the boundary $\partial \mathcal{I}$ is a surface, i.e. a 2-manifold without boundary. In Section 3 we define a "foreground graph" $G_{\mathcal{I}}$, a "background graph" $G_{\mathcal{I}^{c}}$, and a "boundary graph" $G_{\partial \mathcal{I}}$ associated with $\mathcal{I}$, the complement of $\mathcal{I}$, and $\partial \mathcal{I}$, respectively. These graphs play a critical role in what is to follow.

We begin by briefly mentioning a current application: The human cerebral cortex, when viewed as closed at the brain stem, is topologically a sphere and divides the cranial cavity into two regions, interior and exterior. Magnetic resonance imaging (MRI) of the brain can distinguish between tissue that is interior to the cerebral cortex and tissue that is exterior to it. Because of the finiteness of resolution, an MR image can be interpreted as a digital image, with the cubes representing tissue interior to the cerebral cortex. The boundary of the digital image may be taken as an approximation of the cerebral cortex itself. 
Although topologically spherical, the cerebral cortex is densely folded. Thus, the finite resolution, as well as signal noise, may lead to "detection" of topological "handles" that do not actually exist. It is important to "correct" the topology of the digital image, since the physiological and neurological function of regions of the cerebral cortex, as well as the relationship between the regions, is dictated by the spherical topology rather than just spatial proximity. A number of different strategies are currently used [5], [6].

The strategy of Shattuck and Leahy [6] is fundamentally based on the foreground and background graphs; they conjectured that the digital image boundary is topologically spherical if and only if both foreground and background graphs are trees. In situations where one or both of the graphs are not trees, these graphs serve as very direct guides to their topology-correction algorithm. At the conclusion of the algorithm, both foreground and background graphs are trees and, by their conjecture, the boundary of the resulting digital image is topologically spherical.

The Shattuck-Leahy conjecture was proven and generalized by Abrams et al. in [2] and [3]. A further generalization by the present authors [1], mentioned here as Theorem 1, establishes that the genus of a digital image boundary is bounded below and above by the maximum and sum, respectively, of the cycle ranks of the foreground and background graphs. (It is shown there that these bounds are, in a strong sense, best possible.) In particular, these bounds also prove the Shattuck-Leahy conjecture, since they imply that the genus of the digital image boundary is 0 if and only if both foreground and background graphs have cycle rank 0 , which means precisely that foreground and background graphs are both trees.

If $M$ is a closed surface embedded in $\mathbb{R}^{3}$ with a "height function" $f: M \rightarrow \mathbb{R}$, then there is an associated one-dimensional space called a "Reeb graph." It is known [4] that if $f$ is a Morse function, then the genus of $M$ is precisely the cycle rank of the Reeb graph associated with $f$. We give formal definitions and discuss these ideas further in Section 2 . In our context, the boundary of a digital image $\mathcal{I}$ is a closed surface embedded in $\mathbb{R}^{3}$, the $z$-axis serves as a natural height function, and the associated Reeb graph is topologically equivalent to the boundary graph $G_{\partial \mathcal{I}}$. Nevertheless, this height function is not a Morse function (in fact, it is degenerate everywhere), and thus the cycle rank of $G_{\partial \mathcal{I}}$ may fail to equal the surface's genus. Theorem 4 provide several characterizations for when this failure occurs; in particular, failure occurs precisely when there is inequality in the upper bound of Theorem 1.

Our main results are stated in Section 4. The central result is Theorem 2, which expresses the genus of a digital image boundary in terms of the digital image's foreground, background, and boundary graphs. Specifically, the genus of a digital image boundary is precisely half the sum of the respective cycle ranks of the foreground, background, and boundary graphs. Theorems 2 and 3 are proven in Section 6, after the development in Section 5 .

\section{Morse Functions and Reeb Graphs}

In this manuscript the term surface refers to a subset $M$ of $\mathbb{R}^{3}$ that is compact, connected, and locally homeomorphic to an open disk in $\mathbb{R}^{2}$; in particular, $M$ is orientable and has no boundary. We let $g(M)$ denote the genus of $M$. 
Suppose $M$ is a surface. A function $f: M \rightarrow \mathbb{R}$ is a Morse function if all critical points of $f$ are nondegenerate, i.e. have nonsingular Hessian. Given such a function $f$ on $M$, define an equivalence relation on $M$ as follows: $x \sim y$ if and only if $f(x)=f(y)$ and $x, y$ lie in the same connected component of $f^{-1}(f(x))$. The Reeb graph of $f$ on $M$ is the quotient space associated with this equivalence relation, under the quotient topology. It may be viewed as a combinatorial (multi)-graph by considering the points of the Reeb graph not locally homeomorphic to an open interval in $\mathbb{R}$ as graph-theoretic vertices, and considering the connected components of the complement in the Reeb graph of the set of vertices as graph-theoretic edges.

The cycle rank of any combinatorial (multi)-graph $G=(V, E)$ is the value $|E|-$ $|V|+c(G)$, where $c(G)$ is the number of connected components of $G$; the cycle rank $r(G)$ is the first Betti number of $G$ when $G$ is viewed as a one-dimensional space.

The article [4] shows that, given a Morse function $f$ on a surface $M$, the genus of $M$ is equal to the cycle rank of the Reeb graph of $f$ on $M$. (For convenience, an additional condition imposed on $f$ in [4] is that $f$ is injective on the set of critical points.)

Typically, one thinks of Morse functions as "height" functions, and it is true that in our context of the boundary $\partial \mathcal{I}$ of a digital image $\mathcal{I}$ (defined in Section 3 ), the $z$-coordinate $z: \partial \mathcal{I} \rightarrow \mathbb{R}$ is a very natural height function. Unfortunately, it is not a Morse function. In fact, it is degenerate everywhere; at each point that the Hessian is defined, it is 0. (Also worthy of mention is that there are level sets of the $z$-coordinate with infinitely many critical points.) As a consequence, the associated Reeb graph, which is topologically equivalent to $G_{\partial \mathcal{I}}$, will not necessarily satisfy $r\left(G_{\partial \mathcal{I}}\right)=g(\partial \mathcal{I})$. In Theorem 3 we characterize when $r\left(G_{\partial \mathcal{I}}\right)=g(\partial \mathcal{I})$. We also define a foreground graph associated with the interior of $\partial \mathcal{I}$ and an analogous background graph associated with the exterior of $\partial \mathcal{I}$ and, in Theorem 2, we characterized $g(\partial \mathcal{I})$ in terms of the Reeb graph of $\partial \mathcal{I}$ together with these foreground and background graphs. In so doing, the information of the genus of the surface is recovered, despite the height function not being a Morse function.

\section{Definitions}

We begin by recalling some definitions from [1]. Let $N$ be a fixed positive integer. For any triplet of integers $(i, j, k) \in\{1,2, \ldots, N\}^{3}$, define $v_{i, j, k}$ to be the closed Euclidean unit-cube $\left[i-\frac{1}{2}, i+\frac{1}{2}\right] \times\left[j-\frac{1}{2}, j+\frac{1}{2}\right] \times\left[k-\frac{1}{2}, k+\frac{1}{2}\right] \subseteq \mathbb{R}^{3}$.

For any $A \subseteq\{1,2, \ldots, N\}^{3}$, define the digital pre-image $\mathcal{I}_{A}:=\bigcup_{(i, j, k) \in A} v_{i, j, k}$. We call $\mathcal{I}_{A}$ a digital image if its boundary $\partial \mathcal{I}_{A}$ is a surface, and also none of $i, j$, or $k$ equals 1 or $N$. We define $\mathcal{I}_{A}^{C}$ as $\mathcal{I}_{A^{C}}$, where $A^{C}:=\{1,2, \ldots, N\}^{3} \backslash A$. When there is no confusion, we write $\mathcal{I}$ in place of $\mathcal{I}_{A}$.

For each $k \in\{1,2, \ldots, N\}$, the $k$ th level is $L_{k}:=\bigcup_{i, j \in\{1,2, \ldots, N\}} v_{i, j, k}$, and the $(k, k+$ 1)th sheet is $S_{k, k+1}:=L_{k} \cap L_{k+1}$. Associated to a digital pre-image $\mathcal{I}$ is the (multi)-graph $G_{\mathcal{I}}$ with vertex set $V_{\mathcal{I}}$ and edge set $E_{\mathcal{I}}$ defined as follows: For each $k \in\{1,2, \ldots, N\}$, we declare each connected component of $\mathcal{I} \cap L_{k}$ to be a vertex in $V_{\mathcal{I}}$. For any two vertices $u$ and $v$ on adjacent levels, say $L_{k}$ and $L_{k+1}$, we declare each connected component of $u \cap v \subseteq S_{k, k+1}$ to be an edge in $E_{\mathcal{I}}$ whose graph-theoretic endpoints are $u$ and $v$. When referring to a vertex $u \in V_{\mathcal{I}}$ or an edge $\varepsilon \in E_{\mathcal{I}}$, context will dictate whether we are viewing $u$ or $\varepsilon$ as Euclidean subsets, i.e. subsets of $\mathbb{R}^{3}$, or as discrete graph-theoretic objects. If $\mathcal{I}$ is a digital image, we refer to $G_{\mathcal{I}}$ as the "foreground graph" and to $G_{\mathcal{I}^{c}}$ as 
the "background graph," and to their respective vertices and edges correspondingly. We refer to those vertices in $G_{\mathcal{I}^{c}}$ which intersect the line $(1,1, z) \in \mathbb{R}^{3}$ as spine vertices, and to the subgraph of $G_{\mathcal{I}^{c}}$ induced by the spine vertices as the spine.

Also associated to a digital image $\mathcal{I}$ is the (multi)-graph $G_{\partial \mathcal{I}}$ with vertex set $V_{\partial \mathcal{I}}$ and edge set $E_{\partial \mathcal{I}}$ defined as follows: For each sheet $S_{k, k+1}$, we declare the connected components of $\partial \mathcal{I} \cap S_{k, k+1}$ to be vertices of $G_{\partial \mathcal{I}}$. Now let $L_{k}^{\mathrm{o}}$ denote the open $k$ th level, i.e. $L_{k}^{\mathrm{o}}:=L_{k} \backslash\left(S_{k-1, k} \cup S_{k, k+1}\right) \subset \mathbb{R}^{3}$. For each level $L_{k}$, we declare the respective closures of the connected components of $L_{k}^{\mathrm{o}} \cap \partial \mathcal{I}$ to be edges of $G_{\partial \mathcal{I}}$. (It becomes important in Section 5 to note that, as topological subspaces of $\mathbb{R}^{3}$, these edges are cylinders.) The graph-theoretic ends of such an edge are the two vertices of $G_{\partial \mathcal{I}}$ which contain a [one-dimensional] boundary of that edge. We refer to $G_{\partial \mathcal{I}}$ as the "boundary graph," and to its vertices and edges correspondingly. As mentioned above, the graph $G_{\partial \mathcal{I}}$ is topologically equivalent to the Reeb graph of the $z$-coordinate height function on $\partial \mathcal{I}$.

Figure 1 displays several examples of digital images $\mathcal{I}$ and their respective foreground, background, and boundary graphs. Figure 2 displays two adjacent levels and the sheet in between, to illustrate vertices and edges of foreground, background, and boundary graphs further.
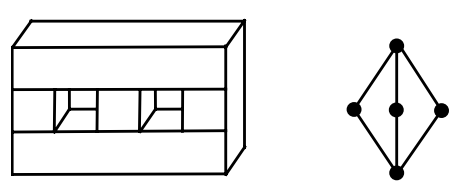

$G_{\mathcal{I}}$
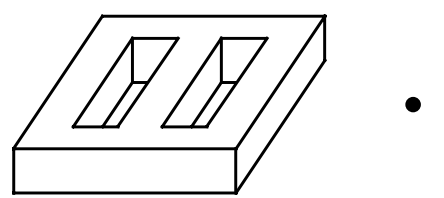

$$
G_{\mathcal{I}}
$$

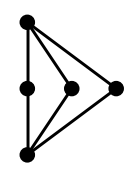

$G_{\mathcal{I}^{c}}$

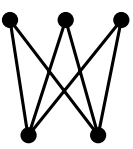

$G_{\mathcal{I}}$

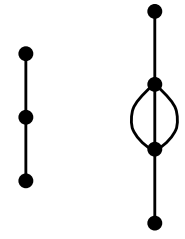

$G_{\mathcal{I}^{c}}$

$G_{\partial \mathcal{I}}$

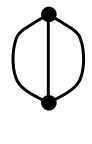

$G_{\partial \mathcal{I}}$

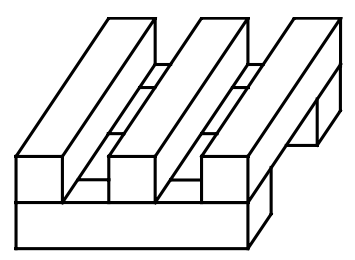

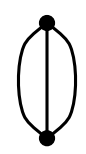

$G_{\mathcal{I}^{c}}$

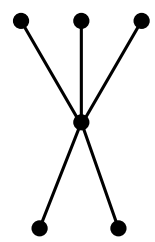

$G_{\partial \mathcal{I}}$

Fig. 1. Some digital images with their respective foreground, background, and boundary graphs. (Note that background vertices corresponding to levels which have no foreground vertices have been deleted from the graphs $G_{\mathcal{I}^{C}}$.) 


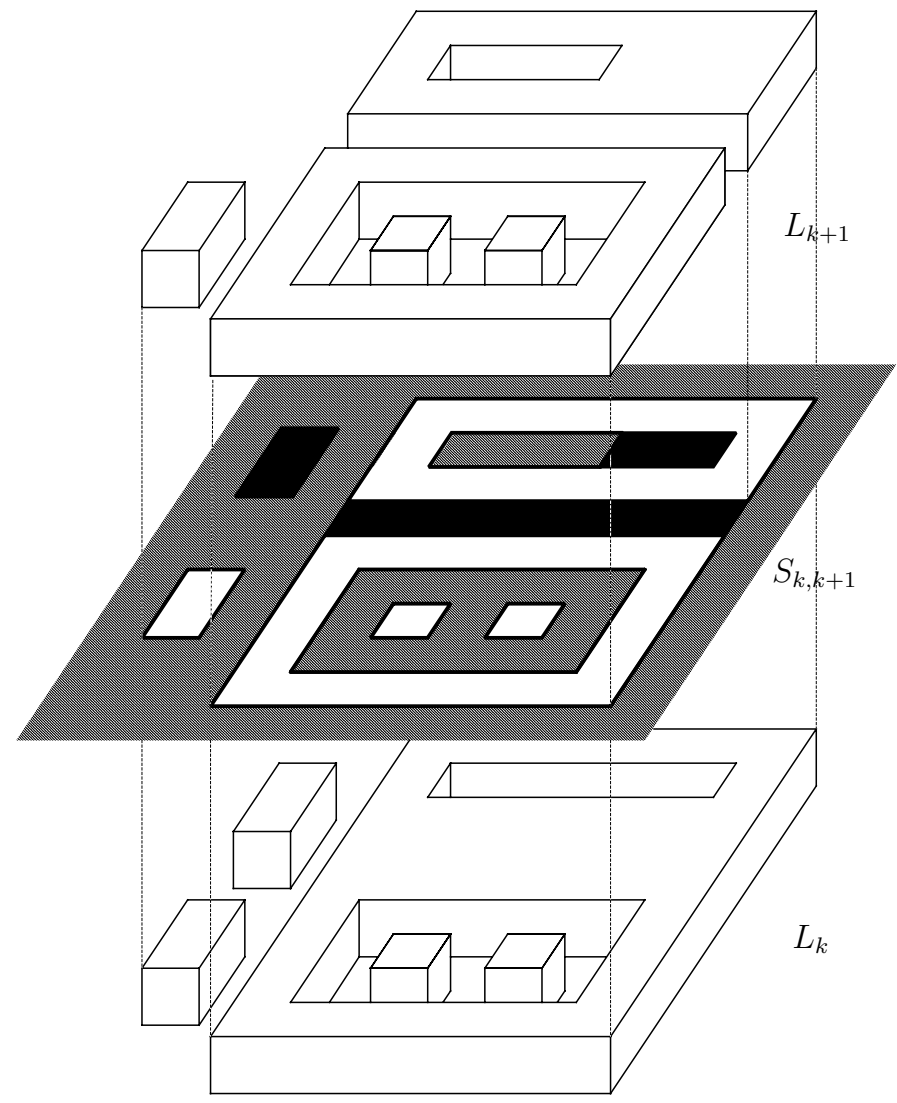

Fig. 2. Two adjacent levels, with the sheet between. In this sheet, the five connected components of white are foreground edges, the three shaded connected components are background edges, and the seven connected components of black are boundary vertices. The degrees of the boundary vertices in this sheet are $1,2,2,2,2,2,3$.

\section{Results}

Let $\mathcal{I}$ be a digital image. Recall that the genus $g(\partial \mathcal{I})$ of the surface $\partial \mathcal{I}$ is the first Betti number of $\mathcal{I}$ and that, for any graph $G=(V, E)$, the cycle rank $r(G):=|E|-|V|+c(G)$ is the first Betti number of $G$; in particular, $r(G)=0$ if and only if $G$ is acyclic. Also note that each of $G_{\mathcal{I}}, G_{\mathcal{I}^{c}}$, and $G_{\partial \mathcal{I}}$ is connected. The following result, Theorem 1, was proved in [1].

Theorem 1. For any digital image $\mathcal{I}$,

$$
\max \left\{r\left(G_{\mathcal{I}}\right), r\left(G_{\mathcal{I}^{c}}\right)\right\} \leq g(\partial \mathcal{I}) \leq r\left(G_{\mathcal{I}}\right)+r\left(G_{\mathcal{I}^{c}}\right) .
$$

Moreover, this is best possible in the sense that, for any nonnegative integers $a, b$, and $c$ such that $\max \{a, b\} \leq c \leq a+b$, there exists a standard digital image $\mathcal{I}$ such that $r\left(G_{\mathcal{I}}\right)=a, r\left(G_{\mathcal{I}^{c}}\right)=\bar{b}$, and $g(\partial \mathcal{I})=c$. 
It should be pointed out that Theorem 1 implies the Shattuck-Leahy conjecture that $\partial \mathcal{I}$ is topologically a sphere if and only if both $G_{\mathcal{I}}$ and $G_{\mathcal{I}^{c}}$ are trees.

Incorporating data from the graph $G_{\partial \mathcal{I}}$ allows for an exact expression of $g(\partial \mathcal{I})$ in terms of $G_{\mathcal{I}}, G_{\mathcal{I}^{c}}$, and $G_{\partial \mathcal{I}}$; Theorem 2 following is the central result of this manuscript.

Theorem 2. For any digital image $\mathcal{I}$,

$$
g(\partial \mathcal{I})=\frac{1}{2}\left(r\left(G_{\mathcal{I}}\right)+r\left(G_{\mathcal{I}^{c}}\right)+r\left(G_{\partial \mathcal{I}}\right)\right) .
$$

Theorem 2 is proved in Section 6.

As pointed out in Section 2, the genus of a surface is the cycle rank of its Reeb graph relative to any Morse function. In our situation with $\partial I$, where the $z$-coordinate height function is not a Morse function, the following result-Theorem 3-characterizes how much the genus of $\partial \mathcal{I}$ differs from the cycle rank of $G_{\partial \mathcal{I}}$, this graph being topologically equivalent to the Reeb graph of the surface $\partial \mathcal{I}$ relative to the $z$-coordinate height function.

Let $V_{\mathcal{I}}$ and $E_{\mathcal{I}}$ denote the vertex set and edge set, respectively, of $G_{\mathcal{I}}$, let $V_{\mathcal{I}}^{k}$ denote the set of vertices of $G_{\mathcal{I}}$ lying in level $L_{k}$, let $E_{\mathcal{I}}^{k, k+1}$ denote the set of edges of $G_{\mathcal{I}}$ lying in sheet $S_{k, k+1}$, and let $G_{\mathcal{I}}^{k, k+1}$ denote the induced subgraph of $G_{\mathcal{I}}$ on vertex set $V_{\mathcal{I}}^{k} \cup V_{\mathcal{I}}^{k+1}$. Define $V_{\mathcal{I}^{c}}, E_{\mathcal{I}^{c}}, V_{\mathcal{I}^{c}}^{k}, E_{\mathcal{I}^{c}}^{k, k+1}, G_{\mathcal{I}^{c}}^{k, k+1}$ and $V_{\partial \mathcal{I}}, E_{\partial \mathcal{I}}, V_{\partial \mathcal{I}}^{k, k+1}, E_{\partial \mathcal{I}}^{k}$ analogously.

Theorem 3. For any digital image $\mathcal{I}$,

$$
g(\partial \mathcal{I})=r\left(G_{\partial \mathcal{I}}\right)+\frac{1}{2}\left(\sum_{k=1}^{N-1} r\left(G_{\mathcal{I}}^{k, k+1}\right)+\sum_{k=1}^{N-1} r\left(G_{\mathcal{I}^{c}}^{k, k+1}\right)\right) .
$$

Theorem 3 is also proved in Section 6.

The next result, Theorem 4, is a corollary of Theorems 2 and 3, and characterizes when the genus of $\partial I$ is not equal to $r\left(G_{\partial \mathcal{I}}\right)$.

Theorem 4. For any digital image $\mathcal{I}$, the following statements are equivalent:

1. It is true that $g(\partial \mathcal{I})=r\left(G_{\partial \mathcal{I}}\right)$.

2. For all $k, G_{\mathcal{I}}^{k, k+1}$ and $G_{\mathcal{I}^{c}}^{k, k+1}$ are acyclic.

3. It is true that $g(\partial \mathcal{I})=r\left(G_{\mathcal{I}}\right)+r\left(G_{\mathcal{I}^{c}}\right)$; i.e. equality holds in the upper bound of Theorem 1.

Proof. The equivalence of statements 1 and 2 follows from Theorem 3 , since $G_{\mathcal{I}}^{k, k+1}$ and $G_{\mathcal{I}^{c}}^{k, k+1}$ are acyclic precisely when $r\left(G_{\mathcal{I}}^{k, k+1}\right)=0$ and $r\left(G_{\mathcal{I}^{c}}^{k, k+1}\right)=0$. The equivalence of statements 1 and 3 follows from the rephrasing of Theorem 2 as $g(\partial \mathcal{I})-r\left(G_{\partial \mathcal{I}}\right)=$ $r\left(G_{\mathcal{I}}\right)+r\left(G_{\mathcal{I}^{c}}\right)-g(\partial \mathcal{I})$.

The next result, Theorem 5, may be thought of as a generalization of the theorem establishing the Shattuck-Leahy conjecture. 
Theorem 5. For any digital image $\mathcal{I}$, the following statements are equivalent:

1. The surface $\partial \mathcal{I}$ is topologically equivalent to a sphere.

2. Each of the graphs $G_{\mathcal{I}}, G_{\mathcal{I}^{c}}$, and $G_{\partial \mathcal{I}}$ is a tree.

3. At least two of the graphs $G_{\mathcal{I}}, G_{\mathcal{I}^{c}}$, and $G_{\partial \mathcal{I}}$ are trees.

Proof. The equivalence of statements 1 and 2 is an immediate consequence of Theorem 2. Of course statement 2 implies statement 3, and we showed in [2] that if $G_{\mathcal{I}}$ and $G_{\mathcal{I}^{c}}$ are both trees then $\partial \mathcal{I}$ is topologically spherical. So all that remains to be shown is that if $G_{\partial \mathcal{I}}$ is a tree and one of $G_{\mathcal{I}}$ and $G_{\mathcal{I}^{c}}$ is a tree, then the other is a tree as well. Indeed, suppose without loss of generality that $G_{\partial \mathcal{I}}$ and $G_{\mathcal{I}^{c}}$ are trees. By Theorem 2 we have $2 g(\partial \mathcal{I})=r\left(G_{\mathcal{I}}\right)$, yet by Theorem 1 we have $r\left(G_{\mathcal{I}}\right) \leq g(\partial \mathcal{I})$, which together imply $r\left(G_{\mathcal{I}}\right)=0$, i.e. $G_{\mathcal{I}}$ is also a tree.

The following result, Proposition 6, is proved in [2]. A multipath is a graph which is a path when multiplicities of edges are ignored.

Proposition 6. Let $\mathcal{I}$ be a digital image. Then every foreground vertex is topologically a ball if and only if $G_{\mathcal{I}^{c}}$ is a multipath of length $N-1$. If this indeed occurs, then $g(\partial \mathcal{I})=r\left(G_{\mathcal{I}}\right)$.

For a digital image in which every foreground vertex is topologically a ball, we have the following simplification of Theorem 3.

Corollary 7. If $\partial \mathcal{I}$ is a digital image in which every foreground vertex is topologically a ball, then

$$
g(\partial \mathcal{I})=r\left(G_{\partial \mathcal{I}}\right)+r\left(G_{\mathcal{I}^{c}}\right)
$$

and, moreover,

$$
r\left(G_{\mathcal{I}^{C}}\right)=\sum_{i=1}^{N-1} r\left(G_{\mathcal{I}^{C}}^{k, k+1}\right)=\sum_{i=1}^{N-1} r\left(G_{\mathcal{I}^{k, k+1}}\right) .
$$

Proof. By Proposition 6 we have here that $g(\partial \mathcal{I})=r\left(G_{\mathcal{I}}\right)$; substituting into Theorem 2 yields the first assertion of this corollary. Also by Proposition 6 we have here that $G_{\mathcal{I}^{c}}$ is a multipath, so its cycle rank is the number of extra edges beyond the number of edges in an underlying path, which yields $r\left(G_{\mathcal{I}^{c}}\right)=\sum_{i=1}^{N-1} r\left(G_{\mathcal{I}^{c}}^{k, k+1}\right)$. The equality $\sum_{i=1}^{N-1} r\left(G_{\mathcal{I}^{C}}^{k, k+1}\right)=\sum_{i=1}^{N-1} r\left(G_{\mathcal{I}}^{k, k+1}\right)$ follows from comparing what we have proven so far with Theorem 3 .

\section{Lemmas}

In this section we prove various lemmas that will be used in Section 6 to prove Theorems 2 and 3 . 


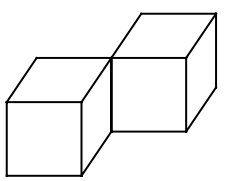

(a)

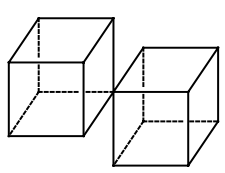

(b)

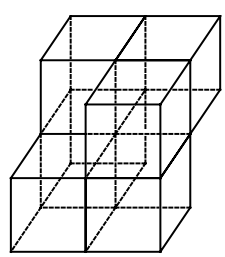

(c)

Fig. 3. The three "forbidden" configurations.

By the Jordan Curve Theorem, any simple, closed curve in $\mathbb{R}^{2}$ partitions $\mathbb{R}^{2}$ into an interior, exterior, and boundary. A simple closed curve $\gamma$ in $\mathbb{R}^{2}$ is called the hull of a subset $\Omega \subseteq \mathbb{R}^{2}$ if $\gamma$ is contained in $\Omega$ and $\Omega$ is also contained in the closure of the interior of $\gamma$. Let $P$ be any plane in $\mathbb{R}^{3}$ perpendicular to the $z$-axis, and let $\pi: \mathbb{R}^{3} \rightarrow P$ denote the orthogonal projection operator.

For topological space $X$, let $\chi(X)$ denote the Euler characteristic of $X$. Recall the Inclusion-Exclusion formula

$$
\chi(X \cup Y)=\chi(X)+\chi(Y)-\chi(X \cap Y)
$$

for any spaces $X, Y$, and the Euler-Poincaré formula

$$
\chi(X)=2-2 g(X)
$$

for any surface $X$.

We begin by pointing out some of the critical implications of our assumption that $\partial \mathcal{I}$ is a surface. In [3] it was shown that the boundary of a digital pre-image is locally homeomorphic to an open disk in $\mathbb{R}^{2}$ if and only if it does not contain anywhere one of the three "forbidden" configurations in Fig. 3. Consequently, as pointed out in [2], each foreground or background vertex $v \in V_{\mathcal{I}} \cup V_{\mathcal{I}^{c}}$ is such that $\partial v$ is itself a surface.

Suppose vertex $v \in V_{\mathcal{I}} \cup V_{\mathcal{I}^{c}}$ is from level $L_{k}$. Note that the respective closures of the connected components of $\partial v \backslash\left(S_{k-1, k} \cup S_{k, k+1}\right)$ are topologically cylinders, and are edges in the boundary graph. We can decompose $\partial v$ as a union of $\partial v \cap S_{k-1, k}, \partial v \cap S_{k, k+1}$, and the cylinders mentioned above. Each of $\partial v \cap S_{k-1, k}, \partial v \cap S_{k, k+1}$ is topologically a disk with punctures, and has Euler characteristic equal to 2 minus the number of cylinders. Since each cylinder has Euler characteristic 0 and has a boundary of Euler characteristic 0 , formulas (1) and (2) show that the genus of $\partial v$ is one less than the number of cylinders, i.e. one less than the number of connected components of $\partial v \backslash\left(S_{k-1, k} \cup S_{k, k+1}\right)$.

Proposition 8. For any digital image $\mathcal{I}$ and each index $k,\left|E_{\partial \mathcal{I}}^{k}\right|=\left|V_{\mathcal{I}}^{k}\right|+\left|V_{\mathcal{I}^{c}}^{k}\right|-1$.

Proof. To each vertex $v \in V_{\mathcal{I}}^{k} \cup V_{\mathcal{I}^{c}}^{k}$ other than the spine vertex in $V_{\mathcal{I}^{c}}^{k}$, we assign the boundary edge $\varepsilon \in E_{\partial \mathcal{I}}^{k}$ for which $\pi(\varepsilon)$ is the hull of $\pi(v)$. This correspondence is a bijection, and the result follows.

Another consequence of $\partial \mathcal{I}$ containing no "forbidden" configuration, also pointed out in [2], is that for each edge $\varepsilon \in E_{\mathcal{I}}^{k, k+1} \cup E_{\mathcal{I}^{c}}^{k, k+1}$, each component of the boundary in 
$S_{k, k+1}$ of $\varepsilon$ is a simple, closed curve; one of these components is the hull of $\varepsilon$ in $S_{k, k+1}$. Let $h(\varepsilon)$ denote the number of "holes" in $\varepsilon$; i.e. $h(\varepsilon)$ is one less than the number of connected components of the boundary in $S_{k, k+1}$ of $\varepsilon$. Since $\varepsilon$ is topologically a disk with $h(\varepsilon)$ punctures, we have $\chi(\varepsilon)=1-h(\varepsilon)$.

Proposition 9. For any digital image $\mathcal{I}$ and each index $k$,

$$
\left|V_{\partial \mathcal{I}}^{k, k+1}\right|=\sum_{\varepsilon \in E_{\mathcal{I}}^{k, k+1}} h(\varepsilon)+\sum_{\varepsilon \in E_{\mathcal{I}^{c}}^{k, k+1}} h(\varepsilon) .
$$

Proof. Let $\gamma$ be a component of the boundary in $S_{k, k+1}$ of a foreground or background edge $\varepsilon$, but which is not the hull of $\varepsilon$. The curve $\gamma$ must be the hull in $S_{k, k+1}$ of a unique boundary vertex $v \in V_{\partial \mathcal{I}}^{k, k+1}$. The function $\gamma \mapsto v$ gives a bijection between the set of "holes" of edges in $E_{\mathcal{I}}^{k, k+1} \cup E_{\mathcal{I}^{c}}^{k, k+1}$ and the vertices $V_{\partial \mathcal{I}}^{k, k+1}$, so the result follows.

For any graph $G$, let $c(G)$ denote the number of connected components in $G$.

Proposition 10. For any digital image $\mathcal{I}$ and each index $k$,

$$
c\left(G_{\mathcal{I}}^{k, k+1}\right)=\sum_{\varepsilon \in E_{\mathcal{I}^{C}}^{k, k+1}} h(\varepsilon) .
$$

Proof. Let $H$ denote a connected component of $G_{\mathcal{I}}^{k, k+1}$, let $\Omega$ denote the union, as Euclidean subsets, of all of the foreground vertices and foreground edges in $H$, and let $\bar{\gamma}$ denote the hull of $\pi(\Omega)$. The curve $\bar{\gamma}$ is equal to $\pi(\gamma)$ for a unique curve $\gamma$ in $S_{k, k+1}$. The curve $\gamma$ is necessarily a connected component of the boundary in $S_{k, k+1}$ of some background edge $\varepsilon \in E_{\mathcal{I}^{c}}^{k, k+1}$, but it cannot be the hull of $\varepsilon$ since it is in the boundary of a foreground graph component. The correspondence $H \mapsto \gamma$ is a bijection between components of $G_{\mathcal{I}}^{k, k+1}$ and "holes" of edges in $E_{\mathcal{I}^{c}}^{k, k+1}$, and the result is established.

Proposition 11. For any digital image $\mathcal{I}$ and each index $k$,

$$
c\left(G_{\mathcal{I}^{c}}^{k, k+1}\right)=1+\sum_{\varepsilon \in E_{\mathcal{I}}^{k, k+1}} h(\varepsilon) .
$$

The proof of Proposition 11 is analogous to the proof of Proposition 10, the only difference in the argument being consideration of the connected component of $G_{\mathcal{I}^{c}}^{k, k+1}$ which contains vertices of the spine.

Proposition 12. For any digital image $\mathcal{I}$ and each index $k,\left|V_{\partial \mathcal{I}}^{k, k+1}\right|=c\left(G_{\mathcal{I}}^{k, k+1}\right)+$ $c\left(G_{\mathcal{I}^{C}}^{k, k+1}\right)-1$.

Proposition 12 is an immediate corollary of Propositions 9-11. 
Lastly, we mention that, by definition of cycle rank, for any digital image $\mathcal{I}$ and each index $k$,

$$
r\left(G_{\mathcal{I}}^{k, k+1}\right)=\left|E_{\mathcal{I}}^{k, k+1}\right|-\left|V_{\mathcal{I}}^{k}\right|-\left|V_{\mathcal{I}}^{k+1}\right|+c\left(G_{\mathcal{I}}^{k, k+1}\right)
$$

and, similarly,

$$
r\left(G_{\mathcal{I}^{c}}^{k, k+1}\right)=\left|E_{\mathcal{I}^{c}}^{k, k+1}\right|-\left|V_{\mathcal{I}^{c}}^{k}\right|-\left|V_{\mathcal{I}^{c}}^{k+1}\right|+c\left(G_{\mathcal{I}^{c}}^{k, k+1}\right) .
$$

\section{Proof of Theorems 2 and 3}

In this section we prove Theorems 2 and 3.

Proof of Theorem 3. Let $\mathcal{I}$ be a digital image. The assertion of Theorem 3 is that

$$
g(\partial \mathcal{I})=r\left(G_{\partial \mathcal{I}}\right)+\frac{1}{2}\left(\sum_{k=1}^{N-1} r\left(G_{\mathcal{I}}^{k, k+1}\right)+\sum_{k=1}^{N-1} r\left(G_{\mathcal{I}^{c}}^{k, k+1}\right)\right) .
$$

We prove this assertion by first calculating the Euler characteristic of $\partial \mathcal{I}$, and then deducing $g(\partial \mathcal{I})$ using the Euler-Poincaré Theorem.

Since the Euler characteristic of a simple, closed curve is 0 , by the Inclusion-Exclusion formula (1) we have that

$$
\chi(\partial I)=\sum_{v \in V_{\partial \mathcal{I}}} \chi(v)+\sum_{\varepsilon \in E_{\partial \mathcal{I}}} \chi(\varepsilon) .
$$

Since each boundary edge $\varepsilon$, as a Euclidean subset, is topologically a cylinder and thus has $\chi(\varepsilon)=0$, we have

$$
\chi(\partial I)=\sum_{v \in V_{\partial \mathcal{I}}} \chi(v) .
$$

We calculate the right-hand side of (5) sheet-by-sheet.

Temporarily fix $k ; S_{k, k+1}$ is a closed disk, so $\chi\left(S_{k, k+1}\right)=1$. Note that the complement in $S_{k, k+1}$ of the union of the interiors of the foreground and background edges is precisely the union of the boundary vertices in $S_{k, k+1}$ (and the hull of $S_{k, k+1}$ ). Applying (1) and keeping in mind that the Euler characteristic of a simple, closed curve is 0 , we therefore have

$$
\chi\left(\partial \mathcal{I} \cap S_{k, k+1}\right)=1-\sum_{\varepsilon \in E_{\mathcal{I}}^{k, k+1}} \chi(\varepsilon)-\sum_{\varepsilon \in E_{\mathcal{I}}^{k, k+1}} \chi(\varepsilon),
$$

since the union of all sets mentioned in the above displayed equation forms $S_{k, k+1}$. As discussed at the beginning of Section 5, each background edge and foreground edge $\varepsilon$ is topologically a punctured sphere, so has $\chi(\varepsilon)=1-h(\varepsilon)$, where $h(\varepsilon)$ is the number of holes in $\varepsilon$. Thus, we have

$$
\begin{aligned}
\chi\left(\partial \mathcal{I} \cap S_{k, k+1}\right) & =1-\sum_{\varepsilon \in E_{\mathcal{I}}^{k, k+1}}[1-h(\varepsilon)]-\sum_{\varepsilon \in E_{\mathcal{I} C}^{k, k+1}}[1-h(\varepsilon)] \\
& =1-\left|E_{\mathcal{I}}^{k, k+1}\right|-\left|E_{\mathcal{I}^{c}}^{k, k+1}\right|+\sum_{\varepsilon \in E_{\mathcal{I}}^{k, k+1}} h(\varepsilon)+\sum_{\varepsilon \in E_{\mathcal{I} C}^{k, k+1}} h(\varepsilon) .
\end{aligned}
$$


Using Propositions 10 and 11, and then Proposition 12, we can rewrite this as

$$
\begin{aligned}
\chi\left(\partial \mathcal{I} \cap S_{k, k+1}\right) & =-\left|E_{\mathcal{I}}^{k, k+1}\right|-\left|E_{\mathcal{I}^{c}}^{k, k+1}\right|+c\left(G_{\mathcal{I}}^{k, k+1}\right)+c\left(G_{\mathcal{I}^{c}}^{k, k+1}\right) \\
& =2\left|V_{\partial \mathcal{I}}^{k, k+1}\right|-\left|E_{\mathcal{I}}^{k, k+1}\right|-\left|E_{\mathcal{I}^{c}}^{k, k+1}\right|-c\left(G_{\mathcal{I}}^{k, k+1}\right)-c\left(G_{\mathcal{I}^{c}}^{k, k+1}\right)+2 .
\end{aligned}
$$

By Proposition 8, we have

$$
\sum_{v \in V_{\partial \mathcal{I}}^{k, k+1}} \operatorname{degree}(v)=\left|E_{\partial \mathcal{I}}^{k}\right|+\left|E_{\partial \mathcal{I}}^{k+1}\right|=\left|V_{\mathcal{I}}^{k}\right|+\left|V_{\mathcal{I}}^{k+1}\right|+\left|V_{\mathcal{I}^{c}}^{k}\right|+\left|V_{\mathcal{I}^{c}}^{k+1}\right|-2 .
$$

Subtracting and then adding $\sum_{v \in V_{\partial \mathcal{T}}^{k, k+1}} \operatorname{degree}(v)$ to the right-hand side of (6), then applying (7), then simplifying the resulting equation with (3), we obtain

$$
\chi\left(\partial \mathcal{I} \cap S_{k, k+1}\right)=2\left|V_{\partial \mathcal{I}}^{k, k+1}\right|-\sum_{v \in V_{\partial \mathcal{I}}^{k, k+1}} \operatorname{degree}(v)-r\left(G_{\mathcal{I}}^{k, k+1}\right)-r\left(G_{\mathcal{I}^{c}}^{k, k+1}\right) .
$$

Summing $\chi\left(\partial \mathcal{I} \cap S_{k, k+1}\right)$ over all $k$ now yields

$$
\chi(\partial \mathcal{I})=2\left|V_{\partial \mathcal{I}}\right|-2\left|E_{\partial \mathcal{I}}\right|-\sum_{k=1}^{N-1} r\left(G_{\mathcal{I}}^{k, k+1}\right)-\sum_{k=1}^{N-1} r\left(G_{\mathcal{I}^{c}}^{k, k+1}\right) .
$$

Using the Euler-Poincaré formula and the previous equation, and then simplifying, yields

$$
g(\partial \mathcal{I})=\frac{1}{2}(2-\chi(\partial \mathcal{I}))=\left|E_{\partial \mathcal{I}}\right|-\left|V_{\partial \mathcal{I}}\right|+1+\frac{1}{2}\left(\sum_{k=1}^{N-1} r\left(G_{\mathcal{I}}^{k, k+1}\right)+\sum_{k=1}^{N-1} r\left(G_{\mathcal{I}^{c}}^{k, k+1}\right)\right),
$$

and Theorem 3 is proved.

Lastly, we prove Theorem 2.

Proof of Theorem 2. Let $\mathcal{I}$ be a digital image; we need to show that

$$
g(\partial \mathcal{I})=\frac{1}{2}\left(r\left(G_{\mathcal{I}}\right)+r\left(G_{\mathcal{I}^{c}}\right)+r\left(G_{\partial \mathcal{I}}\right)\right) .
$$

By Proposition 12, and summing over all sheets, we have

$$
\left|V_{\partial \mathcal{I}}\right|=\sum_{k=1}^{N-1} c\left(G_{\mathcal{I}}^{k, k+1}\right)+\sum_{k=1}^{N-1} c\left(G_{\mathcal{I}^{c}}^{k, k+1}\right)-(N-1) .
$$

By Proposition 8, and summing over all levels, we have

$$
\left|E_{\partial \mathcal{I}}\right|=\left|V_{\mathcal{I}}\right|+\left|V_{\mathcal{I}^{c}}\right|-N .
$$

Combining these two equations together and rearranging yields

$$
\left|E_{\partial \mathcal{I}}\right|-\left|V_{\partial \mathcal{I}}\right|+1-\left|V_{\mathcal{I}}\right|+\sum_{k=1}^{N-1} c\left(G_{\mathcal{I}}^{k, k+1}\right)-\left|V_{\mathcal{I}^{c}}\right|+\sum_{k=1}^{N-1} c\left(G_{\mathcal{I}^{c}}^{k, k+1}\right)=0 .
$$


We can rearrange the equation in Theorem 3 and apply (3) as follows:

$$
\begin{aligned}
g(\partial \mathcal{I})= & r\left(G_{\partial \mathcal{I}}\right)+\frac{1}{2}\left(\sum_{k=1}^{N-1} r\left(G_{\mathcal{I}}^{k, k+1}\right)+\sum_{k=1}^{N-1} r\left(G_{\mathcal{I}^{c}}^{k, k+1}\right)\right) \\
= & \frac{1}{2}\left(\left|E_{\partial \mathcal{I}}\right|-\left|V_{\partial \mathcal{I}}\right|+1\right) \\
& +\frac{1}{2}\left(\left|E_{\partial \mathcal{I}}\right|-\left|V_{\partial \mathcal{I}}\right|+1+\left|E_{\mathcal{I}}\right|-2\left|V_{\mathcal{I}}\right|+\sum_{k=1}^{N-1} c\left(G_{\mathcal{I}}^{k, k+1}\right)\right. \\
& \left.+\left|E_{\mathcal{I}^{c}}\right|-2\left|V_{\mathcal{I}^{c}}\right|+2+\sum_{k=1}^{N-1} c\left(G_{\mathcal{I}^{c}}^{k, k+1}\right)\right) .
\end{aligned}
$$

Substituting (8) into this yields

$$
\begin{aligned}
g(\partial \mathcal{I})= & \frac{1}{2}\left(\left|E_{\partial \mathcal{I}}\right|-\left|V_{\partial \mathcal{I}}\right|+1\right) \\
& +\frac{1}{2}\left(\left|E_{\mathcal{I}}\right|-\left|V_{\mathcal{I}}\right|+1+\left|E_{\mathcal{I}^{C}}\right|-\left|V_{\mathcal{I}^{C}}\right|+1\right),
\end{aligned}
$$

as desired.

\section{References}

1. L. Abrams and D.E. Fishkind, A genus bound for digital image boundaries, SIAM J. Discrete Math., vol. 19, pp. 807-813, 2005.

2. L. Abrams, D.E. Fishkind, and C.E. Priebe, A proof of the spherical homeomorphism conjecture for surfaces, IEEE Trans. Med. Imag., vol. 21, pp. 1564-1566, Dec. 2002.

3. L. Abrams, D.E. Fishkind, and C.E. Priebe, The generalized spherical homeomorphism theorem for digital images, IEEE Trans. Med. Imag., vol. 23, pp. 655-657, May 2004.

4. K. Cole-McLaughlin, H. Edelsbrunner, J. Harer, V. Natarajan, and V. Pascucci, Loops in Reeb graphs of 2-manifolds, Discrete Comput. Geom., vol. 32, pp. 231-244, 2004.

5. X. Han, C. Xu, U. Braga-Neto, and J. Prince, Topology correction in brain cortex segmentation using a multiscale, graph-based algorithm, IEEE Trans. Med. Imag., vol. 21, pp. 109-121, Feb. 2002.

6. D.W. Shattuck and R.M. Leahy, Automated graph-based analysis and correction of cortical volume topology, IEEE Trans. Med. Imag., vol. 20, pp. 1167-1177, Nov. 2001.

Received November 16, 2005, and in revised form December 29, 2006. Online publication April 17, 2007. 